\title{
C-reactive protein level in plasma and drainage blood depends on the method of anaesthesia and post-operative analgesia after hip surgery
}

\author{
Vitaliy Kolomachenko \\ Department of Traumatology, Anaesthesiology and Military Surgery of the Kharkiv Medical Academy \\ of Postgraduate Education, Kharkiv, Ukraine
}

\begin{abstract}
Background: Serum C-reactive protein (CRP) detects the inflammatory response to surgical trauma in hip surgery. It corresponds to the type of surgery, with a higher level of CRP being found in surgery techniques with greater tissue damage. The aim of our study was to analyze the CRP level in serum and drainage blood after hip surgery in patients with coxarthrosis depending on the method of anaesthesia and post-operative analgesia.

Methods: 65 patients, $58 \pm 12$ years old, were included in the study. All patients underwent hip replacement surgery due to coxarthrosis. Serum CRP was measured before surgery and on the $1^{\text {st }}, 3^{\text {rd }}$ and $7^{\text {th }}$ postoperative days. The CRP level in drainage blood was detected on the $1^{\text {st }}, 2^{\text {nd }}$ and $3^{\text {rd }}$ postoperative days. Patients were divided into six groups according to the techniques of intraoperative anaesthesia and postoperative analgesia.

Results: Before surgery, plasma CRP did not differ significantly among the groups. The smallest increase in plasma CRP was found in patients operated on under regional anaesthesia compared with those operated on under general anaesthesia. Postoperatively, the plasma CRP level was higher in patients treated with the systemic administration of opioids+NSAID, than in patients treated with regional analgesia. A significant increase in CRP levels in drainage blood for three days postoperatively was seen in all groups. In patients operated on under general anaesthesia and receiving systemic opioids+NSAID postoperatively, CRP in drainage blood was higher in comparison with those operated on under regional anaesthesia and treated with regional methods of postoperative analgesia.

Conclusion: C-reactive protein levels in plasma and drainage blood depends on the method of anaesthesia and postoperative analgesia after hip surgery. Regional anaesthesia/analgesia methods limit one's inflammatory response to surgical trauma detected by CRP.
\end{abstract}

Anaesthesiology Intensive Therapy 2018, vol. 50, no 2, 117-121

Key words: C-reactive protein; hip surgery; anaesthesia; analgesia

The serum C-reactive protein (CRP) level indicates inflammatory response to surgical trauma in hip surgery. It has been proven that the serum and drainage CRP level corresponds to type of surgery - a higher level of CRP was found in surgical techniques with more tissue damage [1]. Methods of anaesthesia differ in potency of protection from surgical trauma and nociceptive stimuli. Regional anaesthesia is considered being more effective in such protection compared with general anaesthesia. As it was assumed that the method of anaesthesia may have an influence on inflammatory response, this would be detected by the serum CRP level.

The aim of our study was to analyse the CRP level in serum and drainage blood after hip surgery in patients with coxarthrosis depending on the method of anaesthesia and post-operative analgesia.

\section{METHODS}

The study was approved by the Kharkiv Medical Academy of Postgraduate Education Ethics Committee. Before 
inclusion, informed consent was obtained from all patients. A total of 65 patients were included into the study. All patients underwent hip replacement surgery due to coxarthrosis. CRP levels were measured using the latex-enhanced turbidimetric immunoassay technique (Cobas 6000 Analyser, Roche Diagnostics, Switzerland). The serum CRP level was measured before surgery and on the $1^{\text {st }}, 3^{\text {rd }}$ and $7^{\text {th }}$ postoperative days. The CRP level in drainage blood was measured on the $1 \mathrm{st}$, 2nd and 3rd postoperative days. The patients were randomly assigned to six groups according to the techniques of anaesthesia and postoperative analgesia. Group 1 ( $n=11$ ): spinal anaesthesia was used at the L3-L4 levels with $0.5 \%$ bupivacaine $2.4 \mathrm{~mL}$; postoperatively opioids with NSAID (non-steroid anti-inflammatory drug) were administered. Group $2(n=14)$ : spinal anaesthesia was used at the L3-L4 levels with $0.5 \%$ bupivacaine $2.4 \mathrm{~mL}$; postoperatively paravertebral prolonged analgesia using the catheter technique and $0.25 \%$ bupivacaine $3 \mathrm{~mL}$ per $\mathrm{h}$ were administered. Group $3(n=11)$ : spinal anaesthesia was used the L3-L4 levels with $0.5 \%$ bupivacaine $2.4 \mathrm{~mL}$; postoperatively prolonged epidural analgesia using the catheter technique and $0.125 \%$ bupivacaine $3 \mathrm{~mL}$ per $\mathrm{h}$ were administered. Group $4(n=10)$ : psoas compartment block and ischiadic nerve block were provided with $1 \%$ lidocaine $70 \mathrm{~mL}$ with adjuvants (epinephrine 1:200,000; dexametazone $4 \mathrm{mg}$ ); postoperatively opioids with NSAID were used on demand. Group 5 ( $n=11$ ): intraoperatively paravertebral block with $1 \%$ lidocaine $20 \mathrm{~mL}$ using the catheter technique and an epidural block through the caudal approach with $0.75 \%$ ropivacaine $20 \mathrm{~mL}$ were administered; postoperatively the paravertebral block was prolonged. Group $6(n=8)$ : intraoperatively general intravenous anaesthesia (thiopental and fentanyl and pipecuronium) with tracheal intubation and mechanical ventilation was provided; postoperatively opioids with NSAID were administered.

Results were analyzed statistically using multivariate tests of significance (Sigma-restricted parameterization, effective hypothesis decomposition) with Wilks' lambda. The correlation between the CRP level in serum and in drainage blood was analyzed using the Pearson coefficient (R). Data were presented as a mean $(95 \%$ confidence interval; $95 \% \mathrm{Cl}$ ). $P<0.05$ was adopted as significant.

\section{RESULTS}

Groups did not differ significantly in age, diagnosis or surgery duration. The patients' physical status was ASAII and ASA-III and did not differ significantly between groups. All patients were operated on by the same surgical team, while the surgical conditions and techniques, or blood loss volume did not differ among the groups. There were no postoperative infections detected in any group of patients.
The dynamics of the serum CRP level among all patients

The initial level of CRP before surgery among all patients was $8.91 \mathrm{mg} \mathrm{L}^{-1}$ (95\% Cl: 5.16-12.66). On the first postoperative day, the serum CRP level increased significantly ( $P<0.0001$ vs. initial level) up to $67.55 \mathrm{mg} \mathrm{L}^{-1}(95 \%$ $\mathrm{Cl}$ : 56.72-78.38). Up to the third postoperative day, serum CRP continued to increase ( $P<0.0001$ vs. initial level and vs. $\mathrm{p} / \mathrm{o}$ day 1$)$ and reached $98.59 \mathrm{mg} \mathrm{L}^{-1}(95 \% \mathrm{Cl}$ : 87.69-109.49). Up to the $7^{\text {th }}$ postoperative day, serum CRP among all patients decreased to $28.2 \mathrm{mg} \mathrm{L}^{-1}$ (95\% Cl: 22.31-34.09), being significantly lower compared with the first and third postoperative days ( $P<0.0001$ vs. p/o days 1 and 3 ), but higher than the initial level $(P<0.0001$ vs. initial level).

The dynamics of drainage blood CRP level among all patients

The CRP level in drainage blood from an operated hip joint on the first postoperative day was $14.19 \mathrm{mg} \mathrm{L}^{-1}(95 \% \mathrm{Cl}$ : 9.09-19.30). On the second postoperative day, a significant increase $(P<0.0001$ vs. p/o day 1$)$ in CRP levels was detected in drainage blood up to $45.23 \mathrm{mg} \mathrm{L}^{-1}$ (95\%Cl: 36.48-53.98). Up to the third postoperative day, the drainage blood CRP increased further up to $71.11 \mathrm{mg} \mathrm{L}^{-1}$ (95\%Cl: 61.32-80.90), it was significantly higher compared to the postoperative days 1 and 2 ( $P<0.0001$ vs. $\mathrm{p} / \mathrm{o}$ day $1 ; P=0.00013$ vs. $\mathrm{p} / \mathrm{o}$ day 2$)$.

\section{THE DEPENDENCE OF THE SERUM CRP LEVEL ON THE METHOD OF ANAESTHESIA}

The dynamics of serum CRP in six groups is given in Figure 1.

The initial serum CRP level did not differ among all six groups (blue line). On the first postoperative day an increase in serum CRP in all groups (red line) was detected. However, the most potent rise in serum CRP was found in group 6, where the patients were operated on under general anaesthesia and in whom opioids with NSAID were administered in the postoperative period. A moderate increase in serum CRP was detected in groups 1 and 4, who were operated on under regional anaesthesia (spinal - in group 1 and psoas compartment block + ischiadic nerve block in group 4) and who had been given opioids with NSAID, postoperatively. The smallest increase in serum CRP was seen in groups 2, 3 and 5 , where patients were operated on under regional anaesthesia methods (spinal anaesthesia in groups 2 and 3; paravertebral block + caudal epidural block in group 5), and postoperatively received regional analgesia using the catheter technique (prolonged paravertebral analgesia in groups 2 and 5; prolonged epidural analgesia in group 3).

A further increase in serum CRP was found on the third postoperative day in all groups (green line). The highest level of serum CRP was found in group 6. On the $7^{\text {th }}$ day, postoperatively, the serum CRP level decreased significantly in all six groups compared with postoperative days 1st and 3rd. At this stage, the serum CRP level was significantly higher 


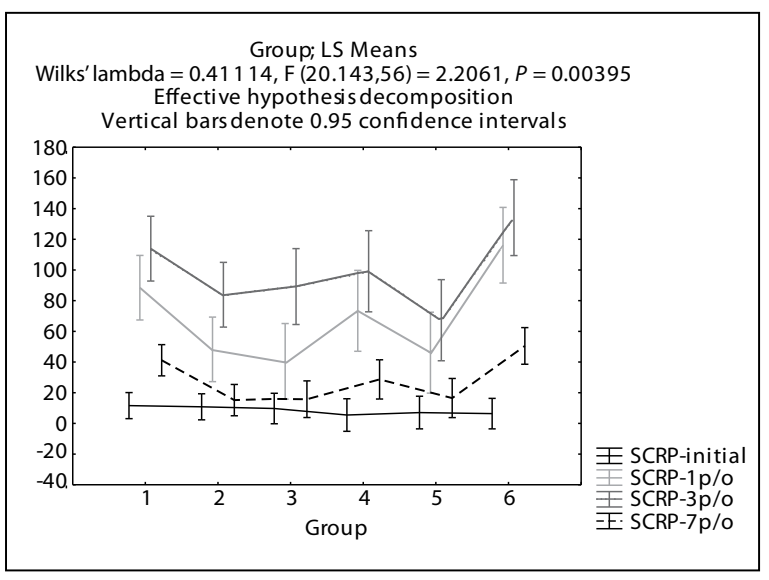

Figure 1. The dynamics of serum CRP levels in groups 1-6 before surgery and on the $1^{\text {st }}, 3^{\text {rd }}, 7^{\text {th }}$ days postoperatively. SCRP - serum C-reactive protein level; initial — before surgery (black line); 1 $\mathrm{p} / \mathrm{o}$ - on the first postoperative day (light gray line); $3 \mathrm{p} / \mathrm{o}-$ on the third postoperative day (dark grey line); $7 \mathrm{p} / \mathrm{o}$ - on the seventh postoperative day (dotted line)

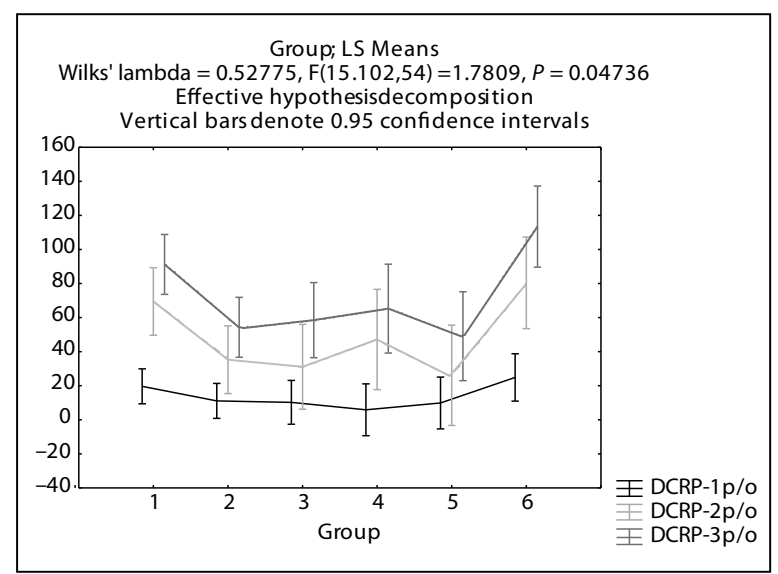

Figure 2. The dynamics of drainage blood CRP levels in groups 1-6 on the $1^{\text {st }}, 2^{\text {nd }}$ and $3^{\text {rd }}$ days postoperatively. DCRP — drainage blood C-reactive protein level; $1 \mathrm{p} / \mathrm{o}$ - on the first postoperative day (black line); 2 p/o - on the second postoperative day (light grey line); 3 $\mathrm{p} / \mathrm{o}$ - on the third postoperative day (dark grey line)

than initially before surgery in groups 1, 4 and 6, while in these three groups opioids with NSAID were administered for postoperative analgesia.

\section{THE DEPENDENCE OF DRAINAGE BLOOD CRP LEVELS ON THE METHOD OF ANAESTHESIA}

The dynamics of drainage blood CRP in six groups is given in Figure 2.

Although on the first postoperative day the CRP level in drainage blood (blue line) was higher in group 6, the difference was not significant among all six groups. On the second postoperative day (red line), the CRP level in drainage blood increased significantly in all groups. The most potent eleva- tion in the drainage blood CRP level was seen in group 6, where the patients were operated on under general anaesthesia and were given opioids with NSAID, postoperatively. A moderate increase was found in groups 1 and 4, in which the patients were operated on under regional anaesthesia and were giving opioids with NSAID, postoperatively. The smallest increase in drainage blood CRP levels was detected in groups 2, 3 and 5, in which the regional methods were administered either intraoperatively or postoperatively. On the third postoperative day, a further increase in drainage blood CRP levels was found. The character of intergroup difference was the same as on the second postoperative day.

\section{THE CORRELATION BETWEEN SERUM AND DRAINAGE BLOOD CRP LEVEL}

Figures 3 and 4 demonstrate the Pearson's correlation between serum and drainage blood CRP levels. On the first postoperative day, a weak positive correlation was found between the levels of CRP in serum and in drainage blood $\left(R^{2}=0.088 ; P=0.0468\right)$.

On the third postoperative day, the positive correlation between the levels of CRP in serum and in drainage blood became stronger and reached a high degree $\left(\mathrm{R}^{2}=0.676 ; P=0.0000\right)$.

\section{DISCUSSION}

C-reactive protein is considered to reflect the development of systemic inflammatory response syndrome. The plasma CRP level in a normal healthy state is $5 \mathrm{mg} \mathrm{L}^{-1}$. In our patients the plasma CRP level was slightly increased. This may be a result of chronic inflammation due to coxarthrosis, which was present in all patients included in the study.

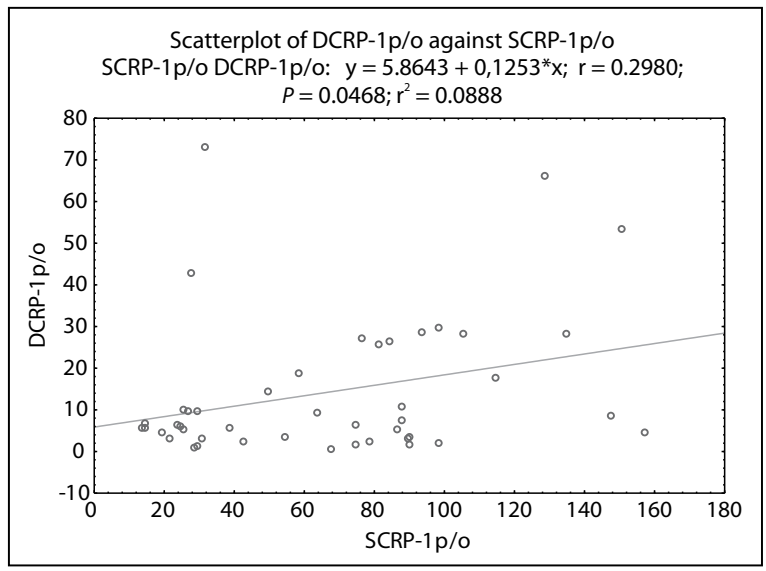

Figure 3. The correlation between the levels of CRP in serum and drainage blood in all patients on the $1^{\text {st }}$ postoperative day. SCRP$1 \mathrm{p} / \mathrm{o}$ - serum C-reactive protein level on the first postoperative day; DCRP-1p/o - drainage blood C-reactive protein level on the first postoperative day 


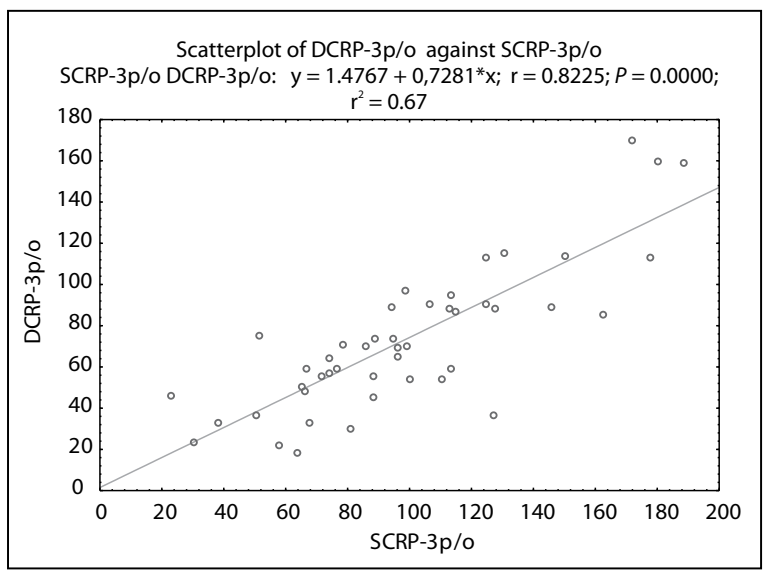

Figure 4. The correlation between the levels of CRP in serum and drainage blood in all patients on the $3^{\text {rd }}$ postoperative day. SCRP$3 \mathrm{p} / \mathrm{o}$ - serum C-reactive protein level on the third postoperative day; DCRP-3p/o - drainage blood C-reactive protein level on the third postoperative day

The rise in plasma CRP on the first postoperative day was about eight-fold. Up to the third postoperative day, serum CRP continued to increase about eleven-fold compared with initial levels. The plasma CRP increase rate can reach several hundred-fold from the initial preoperative state, especially on the first and second postoperative days [2]. CRP levels of about $100-160 \mathrm{mg} \mathrm{L}^{-1}$ were found after total hip or knee arthroplasty [3-6]. In patients who have undergone hip surgery, the plasma CRP level can detect the severity of surgical trauma of bone tissue depending on the surgery type [1]. Up to the $7^{\text {th }}$ postoperative day, serum CRP among all patients decreased, being about three-fold higher than initial levels.

The factors that can cause an inflammatory response with raised CRP in hip surgery are the following: bone and bone marrow injury [7]; opening of the femoral canal; extruding bone marrow from the medullar cavity and loading the lungs with bone marrow and fat during stem preparation, and inserting an endoprosthetic shaft or a femoral nail. CRP is considered to quantify surgical trauma and to assess the surgical trauma experienced by individual patients. [1]. Determining the plasma CRP level is recommended for the diagnosing of prosthetic joint infection [8]. Kim et al. [9] showed that a high preoperative CRP level $\left(>100 \mathrm{mg} \mathrm{L}^{-1}\right)$, a history of malignancy, a high ASA PS grade, and postoperative ICU admission were predominant risk factors for 1-year mortality after hip surgery in geriatric patients.

The methods of anaesthesia differ in their potency of protection from surgical trauma and nociceptive stimuli. Regional anaesthesia is considered to be more effective in such protection compared with general anaesthesia. We compared serum CRP levels in patients who had undergone hip surgery, dependence on the method of anaesthesia used intraoperatively and postoperative analgesia. Before surgery, the initial level of plasma CRP did not differ significantly between groups. We found a lower increase of plasma CRP in patients operated on under regional anaesthesia compared with those operated under general anaesthesia. Even in the postoperative period, the plasma CRP level was higher in patients given the systemic administration of opioids + NSAID, than in patients provided with regional methods of analgesia.

Sousa et al. [10] found that the total leucocyte count in the synovial fluid offers great negative predictive value in the diagnosis of prosthetic joint infection while the addition of more specific markers such as CRP and adenosine deaminase (ADA) improves the positive predictive value. These authors showed that a CRP $>6.7 \mathrm{mg} \mathrm{L}^{-1}$ in the synovial fluid has diagnostic value in prosthetic joint infection.

We detected CRP levels in drainage blood on the first, second and third postoperative days. In all groups, a significant increase in CRP levels in drainage blood was seen for three days postoperatively. We found the significance of CRP levels in drainage blood to be related tp the method of anaesthesia/analgesia. In patients operated on under general anaesthesia and receiving systemic opioids + NSAID postoperatively, the level of CRP in drainage blood was higher in comparison with those operated on under regional anaesthesia and provided with regional methods for postoperative analgesia. We found a high degree of positive correlation between the level of CRP in serum and in drainage blood on the third postoperative day.

\section{CONCLUSIONS}

1. C-reactive protein levels in plasma and drainage blood depends on the method of anaesthesia and postoperative analgesia after hip surgery.

2. Regional anaesthesia/analgesia methods limit the inflammatory response to surgical trauma detected by CRP.

\section{ACKNOWLEDGEMENTS}

1. Source of funding: none.

2. Conflicts of interest: none.

3. Presentation: preliminary data were presented as an oral presentation at the VIII British-Ukrainian Symposium on Anaesthesiology, Kiev, Ukraine in 2016.

\section{References:}

1. Neumaier M, Metak G, Scherer MA. C-reactive protein as a parameter of surgical trauma: CRP response after different types of surgery in 349 hip fractures. Acta Orthop. 2006; 77(5): 788-790, doi: 10.1080/17453670610013006, indexed in Pubmed: 17068712.

2. Foglar C, Lindsey RW. C-reactive protein in orthopedics. Orthopedics. 1998; 21(6): 687-691, doi: 10.3928/0147-7447-19980601-11, indexed in Pubmed: 9642707.

3. Aalto $\mathrm{K}$, Osterman $\mathrm{K}$, Peltola $\mathrm{H}$, et al. Changes in erythrocyte sedimentation rate and C-reactive protein after total hip arthroplasty. Clin Orthop Relat Res. 1984(184): 118-120, indexed in Pubmed: 6705332. 
4. Niskanen RO, Korkala O, Pammo H. Serum C-reactive protein levels after total hip and knee arthroplasty. J Bone Joint Surg Br.. British volume. 1996; 78-B(3): 431-433, doi: 10.1302/0301-620x. 78 b3.0780431.

5. Okafor B, MacLellan G. Postoperative changes of erythrocyte sedimentation rate, plasma viscosity and C-reactive protein levels after hip surgery. Acta Orthop Belg . 1998; 64(1): 52-56, doi: 9586251.

6. White J, Kelly M, Dunsmuir R. C-reactive protein level after total hip and total knee replacement. J Bone Joint Surg Br. 1998; 80(5): 909-911, doi: 10.1302/0301-620x.80b5.8708.

7. Larsson S, Thelander U, Friberg S. C-reactive protein (CRP) levels after

8. elective orthopedic surgery. Clin Orthop Relat Res. 1992; 275: 237-242, indexed in Pubmed: 1735220.

9. Mühlhofer HML, Pohlig F, Kanz KG, et al. Prosthetic joint infection development of an evidence-based diagnostic algorithm. Eur J Med Res. 2017; 22(1): 8, doi: 10.1186/s40001-017-0245-1, indexed in Pubmed: 28274250.

10. Kim BG, Lee YK, Park HP, et al. C-reactive protein is an independent predictor for 1-year mortality in elderly patients undergoing hip fracture surgery: A retrospective analysis. Medicine (Baltimore). 2016 95(43): e5152, doi: 10.1097/MD.0000000000005152, indexed in Pubmed: 27787371

11. Sousa R, Serrano P, Gomes Dias J, et al. Improving the accuracy of synovial fluid analysis in the diagnosis of prosthetic joint infection with simple and inexpensive biomarkers: $C$-reactive protein and adenosine deaminase. Bone Joint J. 2017; 99-B(3): 351-357, doi: 10.1302/0301-620X.99B3. BJJ-2016-0684.R1, indexed in Pubmed: 28249975.

\section{Corresponding author:}

Vitaliy Kolomachenko

248 Saltovske av, ap. 146

Kharkiv, Ukraine 61171

e-mail:kolomach@ukr.net

Received: 8.08.2017

Accepted: 2.06 .2018 